\title{
Etiological Study of Various Retinal Diseases in Paediatric Population at a Tertiary Eye Centre, Telangana State
}

\author{
Aliya Sultana ${ }^{1}$, Bellaganti Hyndavi² \\ ${ }^{1}$ Department of Ophthalmology, Sarojini Devi Eye Hospital, Hyderabad, Telangana, India. \\ ${ }^{2}$ Department of Ophthalmology, Sarojini Devi Eye Hospital, Hyderabad, Telangana, India.
}

\section{ABSTRACT}

\section{BACKGROUND}

Paediatric retinal diseases are very different from adults, presentation is unique compared to adults, various hereditary and acquired causes of retinal disorders are discussed in our study. Though we have significantly advanced techniques to diagnose, still management of these cases is really challenging to vitreo retinal surgeons. We wanted to study various causes of retinal diseases seen in the paediatric population at a tertiary eye hospital in Telangana state.

\section{METHODS}

All paediatric cases presented to vitreo-retinal department of Sarojini Devi Eye Hospital were studied in detail from electronic medical records to know the various retinal diseases in children. Retrospective study was done. Patients of different age groups who presented from paediatric ophthalmology dept. for indirect ophthalmoscopy were examined in detail. Data of patients who presented to vitreoretinal dept. during June 2016 to June 2018 were collected from the records. All patients underwent detailed ocular and systemic examination, and management was done based on the clinical presentation. 120 patients were referred during the two-year period from paediatric ophthalmology department to retina dept.; age range was between 2 to 15 years; mean age of presentation was 9 years, most of the patients were male.

\section{RESULTS}

All 120 patients were examined in detail and treated based on the clinical presentation, many conditions were congenital; acquired causes were also noted. Results were not favourable due to delayed presentation.

\section{CONCLUSIONS}

Early detection of low vision in children helps to prevent blindness. Regular and frequent screening programmes at schools also help in preventing blindness in children.

\section{KEY WORDS}

Retinal Diseases, Paediatric Cases, RRD (Rhegmatogenous Retinal Detachment) Coloboma, PHPV, Retinoblastoma, ROP
Corresponding Author:

Dr. Aliya Sultana,

H. No. 6-3-1099/1/2/3,

Raj Bhavan Road, Somajiguda,

Hyderabad-500082,

Telangana, India.

E-mail: draliyasultana23@gmail.com

DOI: $10.14260 /$ jemds/2019/760

Financial or Other Competing Interests: None.

How to Cite This Article:

Sultana A, Hyndavi B. Etiological study of various retinal diseases in paediatric population at a tertiary eye centre, Telangana State. J. Evolution Med. Dent. Sci. 2019;8(47):3515-3520, DOI: $10.14260 /$ jemds $/ 2019 / 760$

Submission 22-09-2019,

Peer Review 05-11-2019,

Acceptance 11-11-2019,

Published 25-11-2019. 


\section{BACKGROUND}

Main purpose of our study is to provide general view of various hereditary and acquired retinal diseases encountered by vitreo retinal surgeons. Retinal diseases in infants and children are complex topic, proper examination and management is important in these cases. We have various imaging modalities for confirmation of diagnosis. Paediatric retinal diseases are congenital and acquired. Hereditary retinal diseases are group of disorders affecting different cellular structures or retinal development. Examination of parents or siblings is required in some condition to determine familial involvement particularly when the history is not sufficient. Hereditary diseases are vitreo retinopathies, maculopathies and vasculopathies. All these degenerative disorders can manifest as vitreous haemorrhage, macular oedema or retinal detachment at any age of childhood, usually all these disorders are bilateral in presentation. ${ }^{1}$ Acquired retinal diseases are less in percentage in our study compared to congenital group. Most of the acquired causes were retinal detachment, vascular disorders like vasculitis and coats disease.

We wanted to study the various causes of retinal diseases in paediatric population at a tertiary eye centre, Telangana state.

\section{METHODS}

Retrospective study done at Sarojini Devi Eye Hospital, vitreo-retinal dept. Ethics committee has ruled that approval was not required for the study, consent taken from all patients and their attenders for the study. 120 patients presented to retina dept. during June 2016 to June 2018, were studied in detail to know the causes of retinal diseases in paediatrics. Patients presented to paediatric ophthalmology unit were referred to vitreo-retinal dept., for management were included in our study. Patients in the age group of 1-15 years were included in our study. Patients with any trauma history were excluded.

All patients underwent detailed ocular and systemic examination. BCVA, slit lamp examination, indirect ophthalmoscopy, if required fundus fluorescence angiography, OCT, when media is not clear B Scan. Documentation done in all cases, patients were fundus photography was not possible, in those cases Retcam is used for documentation.

Based upon the clinical manifestation management done, medical management, laser therapy, cryo therapy, anti VEGF, surgical management like pars plana vitrectomy, if tamponade required silicone oil is injected. Systemic steroids were given in 8 cases. Laser therapy in 10 cases. Cryo therapy in 6 cases. Anti VEGF in 5 cases. PPV in 12 cases.

\section{RESULTS}

All patients were followed regularly after management, anatomical and visual outcome is not much favourable in most of our cases due to delayed presentation.

\begin{tabular}{|c|c|}
\hline Congenital Retinal Diseases & No. of Cases \\
\hline PHPV & 5 \\
\hline Ocular cutaneous albinism & 5 \\
\hline Coloboma retino choroid & 6 \\
\hline Coloboma retino choroid associated with RRD & 3 \\
\hline FEVR & 4 \\
\hline Retrolental fibroplasia & 2 \\
\hline Syndromic retinitis pigmentosa & 3 \\
\hline Typical retinitis pigmentosa & 7 \\
\hline Cone rod dystrophy & 5 \\
\hline Macular dystrophy & 9 \\
\hline Leber's hereditary optic neuropathy & 4 \\
\hline Retinoblastoma & 6 \\
\hline Retinal Astrocytoma & 1 \\
\hline Disc coloboma & 2 \\
\hline Hypoplastic Disc & 2 \\
\hline Coats disease & 2 \\
\hline \multicolumn{2}{|c|}{ Retinalis Lipaemia } \\
\hline \multicolumn{2}{|c|}{ 1. Cases with Congenital Retinal Disorders } \\
\hline \multicolumn{2}{|c|}{} \\
\hline
\end{tabular}

\begin{tabular}{|c|c|}
\hline Acquired Retinal Diseases & No. of Cases \\
\hline Myopic RRD & 5 \\
\hline Acquired myopia due to regressed ROP & 2 \\
\hline CNVM & 3 \\
\hline Intermediate uveitis & 2 \\
\hline Retinal Vasculitis & 2 \\
\hline Ocular Toxocariasis & 2 \\
\hline Ocular Toxoplasmosis & 2 \\
\hline Choroidal tubercles & 2 \\
\hline Vitreous haemorrhage due to leukemia & 3 \\
\hline Anaemic Retinopathy & 2 \\
\hline Optic Neuritis & 2 \\
\hline \multicolumn{2}{|c|}{ Table 2. Cases with Acquired Retinal Disorders } \\
\hline
\end{tabular}

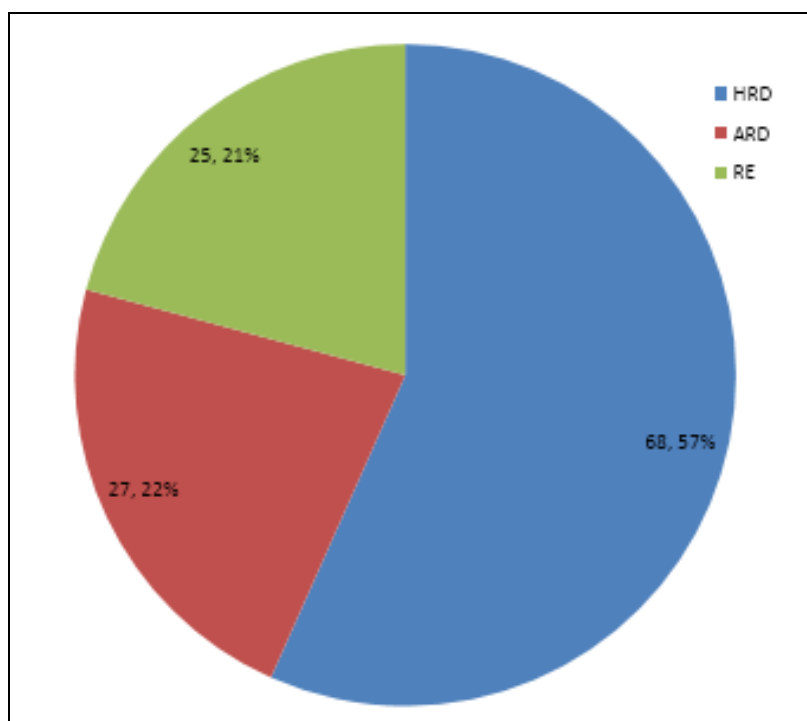

Pie Chart Showing Percentage and Number of Hereditary, Acquired, and Refractive Error Cases

\section{DISCUSSION}

Hereditary retinal diseases were more in percentage compared to acquired retinal disorders in our study. Hereditary conditions were bilateral in presentation, proper clinical and family history is very important. Evaluation of siblings also plays vital role in these cases. Early detection and management can help to prevent blindness in few conditions. Many acquired conditions also needs thorough evaluation for good outcome.

PHPV occurs due to incomplete regression of the embryonic vitreous and hyaloid vasculature. The primary vitreous is formed during the first month of development and contains branches of the hyaloid artery. This hyaloid artery begins to regress during the formation of the avascular 
secondary vitreous at 9 weeks. By the third month, the secondary vitreous, which ultimately forms the adult vitreous, fills most of the developing vitreous cavity. ${ }^{2}$ PHPV cases presented in childhood were almost in phthisical stage, two cases were presented with intra vitreal stalk underwent vitrectomy, one patient had good anatomical and visual outcome, one patient became phthisical after surgery, this patient had micro cornea.

Oculo cutaneous albinism is a condition caused by complete lack or reduction of melanin synthesis in melanocytes leading to hypopigmentation of skin, hair and eyes. Decreased vision is due to foveal hypoplasia and misrouting of the optic nerve fibres. All these patients showed albinotic fundus, patients with poor vision advised OCT to see the foveal contour, abnormal foveal pits noted in most of our patients, spectral domain OCT helps in imaging the foveal morphology. ${ }^{3}$

In our study all our patients were siblings with albinotic fundus, some patients had 6/24 vision in both eyes, some had $6 / 60$ in both eyes. Photophobia was common complaint noted in these patients. Patients with posterior segment colobomas have risk of RRD due to breaks outside coloboma or inside the anomalous tissue within the coloboma or both. Incidence of RRD is $2.4-42 \%$, this can occur in the abnormally dysplastic retina colobomatous retina or extra colobomatous area. ${ }^{4}$

Patients presented with coloboma of posterior segment were treated with barrage laser to the edges of the coloboma as prophylactic treatment to prevent RRD and patients with RRD underwent surgical management, pars plana vitrectomy with silicone oil tamponade. Surgical results in our study were good in 2 cases, in one case anatomical outcome was good but visual outcome was not favourable probably due to secondary glaucoma.

Patients with unilateral coloboma were more common noted in our study compared to bilateral coloboma. In our study 3 cases of coloboma associated with RRD reported, retinal break was not visible. Surgery in coloboma RRD associated with microphthalmos was very difficult to operate due to less visual field. FEVR is usually bilateral and asymmetric. It can present at any age, and the mean age of presentation is 6 years. The main hallmark of FEVR is a vascular peripheral retina with subsequent dragging of the vessels, with or without retinal folds, as well as preretinal, intraretinal, or sub retinal exudation. This may lead to retinal detachment due to traction, exudation, a retinal tear, or a combination of these factors. FEVR may be associated with high myopia, anisometropic amblyopia, and cataract. 5

In our study children with FEVR presented with tractional bands and thickened fibrosed peripheral retina, we noted cases even in young adults presented with sub retinal exudation in macular area and on FFA we could see the dragging of peripheral retinal vessels and avascular retina in the far periphery, patients were managed with photo coagulation and anti VEGF for macular oedema.

The most common factor for development retrolental fibroplasias is not only prematurity, also oxygen therapy to neonate. ${ }^{6}$ Retro lental fibroplasias is one of the cause preschool blindness. Changes identical to retrolental fibroplasias in humans have been produced in animals by exposing them to high oxygen concentration. Oxygen should be ordered for premature infants in a careful and precise manner. Concentrations of over 40 per cent should be avoided whenever possible. Withdrawal to air should be a gradual process. ${ }^{7}$ One 4 years old female baby presented with opaque tissue in periphery with localised retinal detachment, on b scan a mass lesion seen behind lens with few specks of calcification and vacuoles in gel vitreous and localised retinal detachment, advised fundus examination under general anaesthesia, patient not reported back. One male boy underwent vitrectomy with silicone oil tamponade, after removal of silicone secondary IOL implanted, his BCVA is CF 3 mts.

\section{Retinal Dystrophies}

In our study retinal dystrophies were the common disorders noted in our paediatric population, most of the parents need genetic counselling regarding these disorders to prevent blindness. Retinal dystrophies which usually presented in our dept., are non-syndromic, 1 to $2 \%$ patients had syndromic $\mathrm{RP}$, all these patients were of different age groups, some were associated with macular dystrophy and few were associated with myopia. The estimated prevalence of typical RP is 1:5000 while that of syndromic RP is considered less common. These patients were advised low vision aids, early onset cases had low vision compared to young adults. Stargadts macular dystrophy was most commonly noted in our patients. Vitelliform macular dystrophy noted in one female child with vision 6/12 in both eye, one eye showed pre eruptive stage and other eye atrophic stage. In the "classic" presentation of RP, difficulty with dark adaptation begins in adolescence, and visual loss in the mid-peripheral field becomes apparent in young adulthood. However, the age at onset among patients with RP varies widely; thus, some patients develop symptomatic visual loss in early childhood, whereas others can remain relatively asymptomatic until mid-adulthood. The exact age of onset is often difficult to determine, as many patients particularly children are able to compensate for peripheral visual loss. ${ }^{8}$

Mutations in genes involved in ciliary function often but not always result in a syndromic form of RP. The most common ciliopathy is Usher syndrome, which presents with a variable degree of neurosensory hearing loss. Other syndromic form of RP is Bardet-Biedl syndrome; in addition to retinopathy, patients with this syndrome can also present with obesity, postaxial polydactyly, hypogonadism, renal dysfunction, and/or cognitive impairment. ${ }^{8}$ Syndromic RP was noted in 3 patients in our study, two patients were obese with polydactyly, and one patient had RP with deafness.

In LCA, early loss of visual function leads to symptoms that include nystagmus 8 . 3 patients presented with LCA in our study, these patients were advised electrophysiological testing. CME is a common finding in RP patients and is seen in all age groups. The reported prevalence among patients with an autosomal dominant form of RP is relatively high. ${ }^{8}$

In our study CME was highly prevalent in young adult population, in children most of the cases were of atypical presentation. Vascular abnormalities like coats like reactions were also noted in young adults and middle age patients, not in children. Intraocular tumours in children presented to our department were retinoblastoma, one case of astrocytoma also presented, unilateral retinoblastoma was common presentation, bilateral presentation seen in one female child, she had retinoblastoma of type 3 regressive pattern, 
previously treated with chemotherapy developed complicated cataract in one eye. Male predominance noted like other studies. All patients of one eye enucleated for retinoblastoma were followed regularly for other eye fundus examination. Retinoblastoma accounts for $2.5-4 \%$ of all childhood cancers in most developed countries, and a 2- to 3fold higher incidence of tumours of the eye (Majority of which are retinoblastoma in children $<15$ years of age) has been reported in India. India still accounts for nearly one-third of retinoblastoma cases in the Asia Pacific region. ${ }^{9}$ In our study common age of presentation was between 2 to 3 years.

11-year-old boy presented with retinal hamartoma causing full thickness macular hole in one eye, advised surgery, patient mother refused further management. His BCVA was $1 / 60$ at the time of presentation. Coats disease in paediatric patients noted in three cases, male patients, one presented with exudative retinal detachment, drainage of SRF done under general anaesthesia, one patient presented with sub retinal exudation and macular oedema. FFA done in this case, anti VEGF given, and photocoagulation done under general anaesthesia, patient is maintaining 6/60 vision. One patient presented with painful eye, in end stage, prognosis explained to patient's father.

There are two pathological processes which are evident in Coats' disease. ${ }^{8}$ The first consists of a breakdown of the blood-retinal barrier at the endothelial level which causes plasma leakage into the vessel wall and thickening of parts of the vessel wall, becoming necrotic and disorganized. ${ }^{10}$

Second are the abnormal pericytes and endothelial cells in the retinal blood vessels, which subsequently degenerate, causing aneurysms, as well as the closure of vessels leading to ischemia. This causes leakage of lipid-rich exudates into the retina which can lead to changes in the retina, including thickening, cyst formation, or retinal detachment. ${ }^{10}$

Patients presenting with pain, vitreous haemorrhage and anterior segment involvement are commonly seen in Indian eyes. Treatment in the early stages is successful in achieving anatomical stabilization whereas in advanced cases, difficult to treat and results are not favourable. Visual outcome in classic Coats' disease of childhood onset is usually very minimal and frequently, complications will compromise visual function despite an attached retina. ${ }^{11}$

Lipaemia retinalis is a rare condition which can occur in both primary and secondary hyperlipidaemias, it was first described in 1880 by Heyl. Its incidence is directly related to the levels of plasma triglycerides. The characteristic fundus changes include discolouration of retinal blood vessels, which vary from salmon pink to creamy white depending on plasma triglycerides levels. ${ }^{12}$

Two cases reported from children hospital for fundus examination, which revealed white retinal vessels in both eyes, both the patients were infants, advised to avoid breast feeding and start lipid lowering therapy. Two cases never reported back to our department.

Very rarely seen, limited cases noted, need many cases to study about lipaemia retinalis.

Markedly elevated TG in lipemia retinalis might predispose patients to develop potential ocular complications caused by the inner retinal ischemia. Although the literature has shown that fundus lesions of lipemia retinalis might be resolved after appropriate treatment. ${ }^{13}$
Typically, the retinal findings do not occur until the triglyceride level reaches $2500 \mathrm{mg}$ per deciliter. The findings can fluctuate widely from day to day, depending on the triglyceride level. ${ }^{14}$

Acquired retinal disorders reported to our institute were unnoticed rhegmatogenous retinal detachment in both eyes. Patients presented with bilateral RRD, had chronic RRD in both eyes, one with extensive PVR and other with mild PVR. Patients underwent surgery in both eyes had poor anatomical and visual outcome in one eye and other eye showed favourable outcome with some vision in the eye with mild PVR. All cases in our study were myopes.

One young boy presented with myopic foveo schisis, surgery deferred due to absence of foveal detachment. Three patients were male and two were female. One 5-year-old girl patient presented with multiple lattice degeneration and holes; barrage laser done to both eyes done under general anaesthesia.

Prognosis was poor in one girl, both the eyes she had residual RRD inferiorly, due to delayed presentation, other girl under sclera buckling procedure in one eye, maintaining $6 / 24$ vision, another eye had chronic RRD, not operated due to severe PVR.

Complicated cataract and silicone oil keratopathy noted in two patients. Myopia is the common risk factor for RRD in children; prognosis is poor with PVR and high refractive error. Prognosis is poor in old RRD also in large extensive RRD.

Paediatric rhegmatogenous RDs (PRRD) are uncommon $(3 \%-12 \%)$. Most of the studies have shown that males are affected $(70 \%-80 \%)$ more, while others did not show any gender difference. The mean age at presentation is between 9 and 12 years. The management is challenging due to various factors such as anatomic variations, delayed presentation, proliferative vitreoretinopathy (PVR), macula-off detachments with poor presenting visual acuity. These cases should be treated aggressively and with great care as, up to $40 \%$ of eyes, which were treated, had better visual outcomes than the fellow eye. ${ }^{15}$

CNVM noted in three cases, may be due to old healed chorioretinal or traumatic scars and idiopathic was one of the reasons, patients treated with anti VEGF, outcome was good in 2 cases, one case only one line improvement noted due to thick sub retinal membrane. Due to lack of proper history in children it is very difficult to derive aetiology.

Few differences are noted in CNVM of children and adults, in adults usually CNVM is due to myopic macular degeneration or due to age related degeneration, whereas in children could be idiopathic or old traumatic or uveitic scar. ${ }^{16}$ Number of anti VEGF in children required is less compared to adults, according to literature sub foveal was common location, in our study peripapillary CNVM also noted in 10year-old boy may be due to healed neuro retinitis or traumatic scar, this patient presented with thick sub retinal membrane.

CNV in children and adolescents are known to be type 2 membranes, having features of 'classic' CNV on FFA. ${ }^{16}$ Other required retinal diseases were infective and inflammatory condition involving vitreous, optic nerve, retina, retinal vessels, choroid following fever like viral retinitis were commonly noted in children which were treated with systemic steroids and antibiotics. 
One female patient presented with multiple choroidal tubercles, treated with steroids and ATT. Bilateral or unilateral vitreous haemorrhage noted in children of ALL, bleeding disorders, haemorrhagic fever like dengue, these cases were difficult to manage because poor general condition.

Pars plana vitrectomy planned in cases of ALL after paediatrician clearance, due to atrophic retina results were not favourable. Most of the studies showed vasculitis ${ }^{17}$ as the common cause of bilateral vitreous haemorrhage in children, in our study haematological disorders was the common aetiology in non-traumatic conditions.

Acute leukaemias usually present with ocular features of anaemia and thrombocytopenia (In the form of retinal and preretinal haemorrhages, Roth spots, hard exudates, and cotton wool spots). On the other hand, chronic leukaemias present with features of venous stasis and ischemia because of high blood viscosity resulting from increased cell counts. Peripheral retinal nonperfusion and retinal neovascularization are known complications of chronic leukemia, particularly in patients with chronic myeloid leukemia. ${ }^{18}$

Various mechanisms will lead to sluggish blood flow, vascular stasis, capillary dropout, retinal ischemia, neovascularization, and its sequelae in a case of leukemia. These include anaemia, leukocytosis, hyperviscosity, leukoemboli, endothelial damage by toxins released from leukemic cells, and thrombocytopenia. ${ }^{18}$ Proliferative retinopathy in acute leukaemias has been rarely reported. ${ }^{18}$

Anaemic retinopathy was commonly noted post transfusion of blood in children, presented with sudden painless loss of vision in one or both eyes. Clinically fundus examination showed sub hyaloids haemorrhage in the macular area, $\mathrm{Nd}$ :Yag hyaloidotomy tried in one case 10-yearold boy to drain the sub hyaloids haemorrhage. In cases where the hyaloidotomy did not drain, pneumatic displacement tried.

Anaemia is a common problem in children. Most of the complications of severe anaemia are well documented but less literature is available on ocular complications of anaemia. The most common ocular manifestations described are retinal haemorrhages, tortuous retinal veins, cotton-wool exudates and disc oedema. ${ }^{19}$

Optic neuritis was noted in 10-year-old and 13-year-old patients, both the patients are male, bilateral presentation was seen in 13-year-old and unilateral presentation noted in 10 -year-old patient, both of them showed good response to systemic steroids, reoccurrence not occurred in both of them. In children most common aetiology is para infectious optic neuritis.

According to literature bilateral presentation is common compared to unilateral, mean age of presentation is 12.38 (5 -15 age range noted). In contrast to adult optic neuritis, pattern evoked potentials in children with optic neuritis, though abnormal at presentation, are more frequently normal at follow-up (55\% vs $10 \%$ in the adult population), indicating the more benign nature of this disease in the child. ${ }^{20}$ Visual outcome in paediatric optic neuritis or papillitis is always good.

The time of onset of visual recovery was adequately $r$ significant improvement within 24 hours of commencing treatment, whereas $74 \%$ showed a definite improvement within 72 hours. ${ }^{20}$

The pathologic process that affects optic nerve fibers in paediatric optic neuritis is sparse; however, the clinical and electrophysiologic similarities between the acute stages of adult and paediatric optic neuritis, suggest that demyelination plays a prominent role. The more frequent and sometimes more speedy restoration of normal visual evoked potential latency in children than in adults raise the possibility that the process of remyelination is more rapid in children. ${ }^{20}$

Female preponderance, bilateral presentation and papillitis more common in adults compared to children. ${ }^{21}$ Although children are less likely than adults to develop multiple sclerosis after optic neuritis, multiple sclerosis still develops in many cases, especially in those with multiple attacks. It is difficult to understand the clinical picture of optic neuritis in Indian paediatric population.

\section{CONCLUSIONS}

Regular screening programmes in schools will help to identify and manage the cases early to prevent childhood blindness. In our study more percentage of children showed hereditary retinal diseases, percentage of acquired diseases also was quite high; due to delayed presentation the outcome was not favourable. Many non-traumatic causes of retinal diseases in children require early diagnosis. Refractive errors were seen in boys and girls equally; most of them had myopia; correction was done and advised to follow every year for any change in correction. Paediatric retinal diseases are a major topic. In our study uveitis cases were very less. Uveitis in children accounts for $5-10 \%$ of all uveitis population. Because of the prolonged duration and increased risks of complications, paediatric uveitis can lead to significant ocular morbidity. Severe vision loss is found in $25-33 \%$ of paediatric uveitis cases. $^{21}$

\section{REFERENCES}

[1] Say EAT. Genetic paediatric retinal diseases. Journal of paediatric Genetics 2014;3(4):229-41.

[2] Jain TP. Bilateral persistent hyperplastic primary vitreous. Indian Journal of Ophthalmology 2009;57(1):53-4.

[3] Chong GT, Farsiu S, Freedman SF, et al. Abnormal foveal morphology in ocular albinism imaged with spectraldomain optical coherence tomography. Arch Ophthalmol 2009;127(1):37-44.

[4] Suwal B, Paudyal G, Thapa R, et al. A study on pattern of retinal detachment in patients with choroidal coloboma and its outcome after surgery at a tertiary eye hospital in Nepal. Article ID 7390852, Journal of Ophthalmology 2019;2019:5.

[5] Gilmour DF. Familial exudative vitreoretinopathy and related retinopathies. Eye (Lond) 2015;29(1):1-14.

[6] Henry M. Recent advances in retrolental fibroplasia. California Medicine 1954;81(4):272-5. 
[7] Verbakel SK, Van Huet RAC, Boon CJF, et al. Nonsyndromic retinitis pigmentosa. Progress in Retinal and Eye Research 2018;66:157-86.

[8] Singh U, Katoch D, Kaur S, et al. Retinoblastoma: a sixteen-year review of the presentation, treatment, and outcome from a tertiary care institute in Northern India. Ocul Oncol Pathol 2017;4(1):23-32.

[9] Fernandes H, Umashankar T, Richie AJ, et al. Coats' disease - disease of the eye rarely encountered by pathologists. Indian Journal of Pathology and Microbiology 2018;61(1):98-100.

[10] Rishi P, Rishi E, Uparkar M, et al. Coats' disease: an Indian perspective. Indian J Ophthalmol 2010;58(2):11924.

[11] Chaudhur D, Meenakshi R, Ramakrishnan R, et al. Lipaemia retinalis. Delhi Journal of Ophthalmology 2015;26:107-10.

[12] Yin HY, Warman R, Suh EH, et al. Exceptionally elevated triglyceride in severe lipemia retinalis. Int Med Case Rep J 2016;9:333-6.

[13] Rymarz E, Matysik-WoŸniak A, Baltaziak L, et al. Lipemia retinalis - an unusual cause of visual acuity deterioration. Med Sci Monit 2012;18(8):CS72-CS5.
[14] Balakrishnan D. paediatric retinal detachment: an overview. KJO 2018;30(2):87-93.

[15] Rishi P, Gupta A, Rishi E, et al. Choroidal neovascularization in 36 eyes of children and adolescents. Eye (Lond) 2013;27(10):1158-68.

[16] Sudhalkar A, Chhablani J, Rani PK, et al. Bilateral vitreous hemorrhage in children: clinical features and outcomes. J Ophthalmic Vis Res 2015;10(2):139-43.

[17] Kumawat D, Kumar V, Sahay P, et al. Bilateral proliferative retinopathy in B-cell acute lymphoblastic leukemia. Indian J Ophtalmol 2018;66(1):148-51.

[18] Dhir SK, Kaur A, Kaur G, et al. Retinal hemorrhage in severe anemia in children: a case report. International Journal of Community Health and Medical Research 2015;1(1):30-2.

[19] Cassidy L, Taylor D. paediatric optic neuritis. Journal of AAPOS 1999;3(2):68-9.

[20] Khadse R, Ravindran M, Pawar N, et al. Clinical profile and neuroimaging in paediatric optic neuritis in Indian population: a case series. Indian $\mathrm{J}$ Ophthalmol 2017;65(3):242-5.

[21] Majumder PD, Biswas J. paediatric uveitis: an update. Oman Journal of Ophthalmology 2013;6(3):140-50. 